\title{
ON PAIRS OF COEFFICIENTS OF BOUNDED POLYNOMIALS
}

\author{
L. BRICKMAN ${ }^{1}$, Q. I. RAHMAN AND ST. RUSCHEWEYH
}

AbSTRaCt. Given a polynomial of degree at most $n$, we estimate the sum of the moduli of any two of its coefficients in terms of its supremum norm on the unit disk.

1. Let $\mathscr{P}_{n}$ denote the class of polynomials $P$ of degree $\leqslant n$ with

$$
\|P\|=\max \{|P(z)|:|z| \leqslant 1\} \leqslant 1 .
$$

We also set

$$
\begin{aligned}
C_{n}(s, t) & =\max \left\{\left|a_{s}\right|+\left|a_{t}\right|: \sum_{k=0}^{n} a_{k} z^{k} \in \mathscr{P}_{n}\right\}, \quad 0 \leqslant s<t \leqslant n, \\
C_{n} & =\max \left\{C_{n}(s, t): 0 \leqslant s<t \leqslant n\right\} .
\end{aligned}
$$

A classical result of O. Szász [4], originally stated for bounded analytic functions instead of bounded polynomials, implies

$$
\sup C_{n}=4 / \pi
$$

as well as the estimate

$$
C_{n}(s, t)<\sum_{k=0}^{l}\left(\begin{array}{c}
1 / 2 \\
k
\end{array}\right)^{2}, \quad l=\left[\frac{t}{t-s}\right] .
$$

From these one can deduce that there exists a constant $c>0$ such that

$$
C_{n} \leqslant 4 / \pi-c / n^{2}, \quad n \in \mathbf{N} \text {. }
$$

In this note we prove

TheOREM 1. Let $D_{n}=\left(4 / \pi-C_{n}\right) n^{2}, n \in \mathbf{N}$. Then

$$
\varlimsup_{n \rightarrow \infty} D_{n} \leqslant \frac{4 \pi}{3}=4.18 \ldots,
$$

and

$$
\lim _{n \rightarrow \infty} D_{n} \geqslant \frac{(1+\pi \sqrt{8 / 3})^{2}}{8 \pi}=1.49 \ldots
$$

Received by the editors November 2, 1983.

1980 Mathematics Subject Classification. Primary 26C05, 30A10, 30C10, 42A05.

Key words and phrases. Bounded analytic functions, pairs of coefficients, polynomials.

${ }^{1}$ This work was done at Würzburg University and supported by the Deutsche Forschungsgemeinschaft. 
Besides (2), the main tool in our approach is the following estimate of $Q$. I. Rahman [3] (see also L. Brickman and St. Ruscheweyh [1]):

$$
C_{n}(s, t) \leqslant \frac{2}{m} \cot \frac{\pi}{2 m}, \quad 0 \leqslant s<t, s+t \leqslant n, m=\left[\frac{n-s}{t-s}+1\right] .
$$

(6) is known to be best possible in a number of cases; for instance, if $n$ is odd, $s=[n / 2], s+t=n$, and these cases lead to (4). Unfortunately, the exact range of parameters for which (6) is sharp remains unknown. We have only marginal results:

THEOREM 2. (6) is best possible if either $m \in\{2,3,4\}$ or $s \geqslant(m-2)(t-s)$.

Note that (6) cannot be sharp in every case, since then $\lim _{n \rightarrow \infty} C_{n}(s, t)=4 / \pi$ for $s, t$ fixed, which contradicts (2). To determine $C_{n}(s, t)$ in the cases where (6) is not sharp seems to be even harder. It would be interesting to narrow the gap in (4) and (5) and to decide the question of whether or not $\lim _{n \rightarrow \infty} D_{n}$ exists.

2. Proof of Theorem 2. For $m \geqslant 2$ let

$$
P_{2 m-3}(z)=\sum_{k=0}^{2 m-3} A_{k} z^{k}
$$

with

$$
\begin{gathered}
A_{k}=\frac{(-1)^{m+k}}{2 m^{2}}\left[(2 k+3) \cot \frac{(2 m-3-2 k) \pi}{2 m}+\cot \frac{\pi}{2 m}\right], \\
A_{2 m-3-k}=A_{k}, \quad k=0,1, \ldots, m-2 .
\end{gathered}
$$

It is due to Mulholland [2] (compare Rahman [3]) that $\left\|P_{2 m-3}\right\|=1$, and we obviously have

$$
\left|A_{m-2}\right|+\left|A_{m-1}\right|=\frac{2}{m} \cot \frac{\pi}{2 m},
$$

which shows that (6) is sharp for this case.

Now let $n, s, t$ be as in (6) such that $s \geqslant(m-2)(t-s)$. Then

$$
Q(z)=z^{s-(m-2)(t-s)} P_{2 m-3}\left(z^{t-s}\right)
$$

is of degree

$$
s+(m-1)(t-s)=s+[(n-s) /(t-s)](t-s) \leqslant n .
$$

Furthermore, $\|Q\|=1$ and the coefficients with index $s$ and $t$, respectively, add up to the expected sum.

Next define the polynomials

$$
\begin{aligned}
& R_{2}(z)=\frac{1}{2}(1-z) \\
& R_{3}(z)=\frac{1}{3 \sqrt{3}}\left(-2+4 z+z^{2}\right) \\
& R_{4}(z)=\alpha+\beta-(\alpha+3 \beta) z+(\alpha-3 \beta) z^{2}-(\alpha-\beta) z^{3}
\end{aligned}
$$


with $\alpha=1 / 4, \beta=1 / \sqrt{32}$. A simple verification yields

$$
R_{m}\left(e^{i \theta}\right)^{2}=1-\frac{2}{m^{3}}\left(\frac{\sin (m / 2 \theta)}{\sin (\theta / 2)}\right)^{2}, \quad \theta \in \mathbf{R}, m=2,3,4
$$

hence $\left\|R_{m}\right\|=1$. Furthermore,

$$
\left|R_{m}(0)\right|+\left|R_{m}^{\prime}(0)\right|=\frac{1}{2 m} \cot \frac{\pi}{2 m}, \quad m=2,3,4,
$$

so (6) is sharp for $s=0, t=1, n \in\{1,2,3\}$.

In the general cases $m=2,3,4$, the extreme polynomials are $z^{s} R_{m}\left(z^{t-s}\right) \in \mathscr{P}_{n}$. This completes the proof of Theorem 2.

3. Proof of Theorem 1. (4) follows from Theorem 2. In fact, for $m=2,3, \ldots$ we have

$$
C_{2 m-2} \geqslant C_{2 m-3} \geqslant C_{2 m-3}(m-2, m-1)=\frac{2}{m} \cot \frac{\pi}{2 m} .
$$

Using the expansion

$$
\frac{2}{x} \cot \frac{\pi}{2 x}=\frac{4}{\pi}-\frac{\pi}{3 x^{2}}+o\left(\frac{1}{x^{2}}\right), \quad x \rightarrow \infty,
$$

(4) follows immediately. In the proof of (5) we make use of the following expansion:

$$
\sum_{k=0}^{l}\left(\begin{array}{c}
1 / 2 \\
k
\end{array}\right)^{2}=\frac{4}{\pi}-\frac{1}{8 \pi l^{2}}+o\left(\frac{1}{l^{2}}\right), \quad l \rightarrow \infty .
$$

To establish (8) we first observe that, by Parseval's identity,

$$
\sum_{k=0}^{\infty}\left(\begin{array}{c}
1 / 2 \\
k
\end{array}\right)^{2}=\frac{1}{2 \pi} \int_{0}^{2 \pi}\left|1+e^{i \theta}\right| d \theta=\frac{1}{\pi} \int_{0}^{2 \pi} \sin \frac{\theta}{2} d \theta=\frac{4}{\pi},
$$

so we must prove

$$
\lim _{j \rightarrow \infty} j^{2} \sum_{k=j}^{\infty}\left(\begin{array}{c}
1 / 2 \\
k
\end{array}\right)^{2}=\frac{1}{8 \pi}
$$

Using Stirling's formula

$$
n !=(n / e) \sqrt[n]{2 \pi n} e^{\theta / n}
$$

for a certain $\theta \in(0,1 / 4)$, we obtain

$$
\left(\begin{array}{c}
1 / 2 \\
k
\end{array}\right)^{2}=\frac{1}{k^{2}(k-1)} \frac{1}{4 \pi} e^{\theta^{\prime} /(k-1)}, \quad \theta^{\prime} \in\left(-1, \frac{1}{4}\right) .
$$

Therefore,

$$
\frac{1}{4 \pi} e^{-1 /(j-1)} \sum_{k=j}^{\infty} \frac{j^{2}}{k^{3}} \leqslant j^{2} \sum_{k=j}^{\infty}\left(\begin{array}{c}
1 / 2 \\
k
\end{array}\right)^{2} \leqslant \frac{1}{4 \pi} e^{1 /\{4(j-1)\}} \sum_{k=j}^{\infty} \frac{j^{2}}{(k-1)^{3}} .
$$


Using the integral criterion, we immediately deduce

$$
\lim _{j \rightarrow \infty} \sum_{k=j}^{\infty} \frac{j^{2}}{k^{3}}=\lim _{j \rightarrow \infty} \sum_{k=j}^{\infty} \frac{j^{2}}{(k-1)^{3}}=\frac{1}{2},
$$

which implies (9).

In order to estimate $C_{n}(s, t), 0 \leqslant s<t \leqslant n$, we note that

$$
C_{n}(s, t)=C_{n}(n-t, n-s),
$$

since $P$ belongs to $\mathscr{P}_{n}$ if and only if $\tilde{P}(z):=z^{n} \overline{P(1 / \bar{z})}$ does; therefore, we can confine ourselves to the cases $s+t \leqslant n$. Here we have two different estimates coming from (2) and (6), namely

$$
C_{n}(s, t) \leqslant\left\{\begin{array}{l}
\sum_{k=0}^{t}\left(\begin{array}{c}
1 / 2 \\
k
\end{array}\right)^{2}=F(t), \\
\frac{2}{n-t+2} \cot \frac{\pi}{2(n-t+2)}=G(t) .
\end{array}\right.
$$

(Note that in both cases the estimates for $C_{n}(t-1, t)$ are the largest among those for $C_{n}(s, t), 0 \leqslant s<t$.) Let

$$
\beta=(1+\pi \sqrt{8 / 3})^{-1} .
$$

From the obvious monotonicities of $F$ and $G$, we get, with $t_{n}=[\beta n]$,

$$
\begin{array}{ll}
F(t) \leqslant F\left(t_{n}\right), & t \leqslant t_{n}, \\
G(t) \leqslant G\left(t_{n}\right), & t \geqslant t_{n} .
\end{array}
$$

This shows that

$$
C_{n} \leqslant \max _{t} \min \{F(t), G(t)\} \leqslant \max \left\{F\left(t_{n}\right), G\left(t_{n}\right)\right\} .
$$

The expansions (7) and (8) yield

$$
\begin{aligned}
& F\left(t_{n}\right)=\frac{4}{\pi}-\frac{1}{8 \pi \beta^{2} n^{2}}+o\left(\frac{1}{n^{2}}\right) \\
& G\left(t_{n}\right)=\frac{4}{\pi}-\frac{\pi}{3(1-\beta)^{2} n^{2}}+o\left(\frac{1}{n^{2}}\right) .
\end{aligned}
$$

Since $8 \pi \beta^{2}=3(1-\beta)^{2} / \pi$, we get

$$
C_{n} \leqslant \frac{4}{\pi}-\frac{(1+\pi \sqrt{8 / 3})^{2}}{8 \pi} \frac{1}{n^{2}}+o\left(\frac{1}{n^{2}}\right),
$$

which corresponds to (5).

\section{REFERENCES}

1. L. Brickman and St. Ruscheweyh, On bound preserving multipliers for $H(\mathbf{D})$ and applications to polynomials, preprint, 1983.

2. H. P. Mulholland, On two extremum problems for polynomials on the unit disc, J. London Math. Soc. 31 (1956), 191-199. 
3. Q. I. Rahman, Inequalities concerning polynomials and trigonometric polynomials, J. Math. Anal. Appl. 6 (1963), 303-324.

4. O. Szász, Ungleichungen für die Koeffizienten einer Potenzreihe, Math. Z. 1 (1918), 163-183.

Department of Mathematics, State University of New York, Albany, New York 12222

Departement de Mathematiques et de Statistique, UniversitE de Montreal, Montreal h3C 3J7, CANADA

Mathematisches Institut der Universität Würzburg, Am Hubland, D - 8700 Würzburg, West GERMANY 